\title{
Validity of the 6-Minute Walk Test in Patients with End-Stage Lung Diseases Wearing an Oronasal Surgical Mask in Times of the COVID-19 Pandemic
}

\author{
Isabell Anna Just ${ }^{a, b}$ Felix Schoenrath ${ }^{a, b}$ Philipp Passinger ${ }^{a} \quad J^{a l i a ~ S t e i n ~}{ }^{c}$ \\ Dagmar Kemper $^{\mathrm{a}}$ Christoph Knosalla ${ }^{\mathrm{a}, \mathrm{b}}$, d Volkmar Falk $^{\mathrm{a}, \mathrm{b}}$, d, e Jan Knierim ${ }^{\mathrm{a}}$

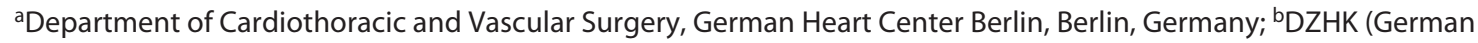 \\ Centre for Cardiovascular Research), Partner Site Berlin, Berlin, Germany; ' Dienstleistungs GmbH, German Heart \\ Center Berlin, Berlin, Germany; ${ }^{d}$ Department of Cardiothoracic Surgery, Charité - Universitätsmedizin Berlin, \\ corporate member of Freie Universität Berlin, Humboldt-Universität zu Berlin, and Berlin Institute of Health, Berlin, \\ Germany; ${ }^{\text {DDepartment }}$ of Health Sciences and Technology, ETH Zurich, Zurich, Switzerland
}

\section{Keywords}

6-minute walk test · Lung transplantation · Advanced pulmonary disease $\cdot$ COVID-19 pandemic

\begin{abstract}
Background and Objectives: The 6-minute walk test (6MWT), as a clinical assessment tool for functional exercise capacity, is an integral component of lung allocation scores (LASs). In times of the coronavirus disease (COVID-19) pandemic, patients underwent 6MWTs wearing a surgical mask in ambulatory care. We investigated the impact of wearing a mask on 6-minute walk distances (6MWDs). Method: 6MWDs of 64 patients with end-stage lung diseases wearing an oronasal surgical mask were retrospectively compared to previously investigated 6MWDs of the same cohort, in a pre-COVID-19 pandemic era, without wearing a mask. Four patients were excluded due to a primary vascular disease, 29 patients due to clinically unstable pulmonary functions, and 1 patient due to a psychiatric disorder. Results: The median age of the patients included was 55 (46-58) years; 15 (48\%) were male. Ten (32.2\%) were on the Eurotransplant lung transplant waiting list with a median LAS of 34.3 (31.9-36.2). Twenty (64.5\%) pa-
\end{abstract}

karger@karger.com www.karger.com/res

Karger

bOPEN ACCESS
(C) 2021 The Author(s)

Published by S. Karger AG, Basel

This is an Open Access article licensed under the Creative Commons Attribution-NonCommercial-4.0 International License (CC BY-NC) (http://www.karger.com/Services/OpenAccessLicense), applicable to the online version of the article only. Usage and distribution for commercial purposes requires written permission. tients had chronic obstructive pulmonary diseases, 7 (22.6\%) had interstitial lung diseases, and 4 (12.9\%) had other endstage lung diseases. The mean 6MWD without versus with wearing a mask was 306.9 (101.9) versus 305.7 (103.8) m, with a mean difference of $-1.19 \mathrm{~m}$ ( $95 \%$ confidence interval -13.4 to 11.03). The observed difference is statistically equivalent to zero $(p<0.001)$. No significant differences in $6 \mathrm{MWDs}$ were observed between the clinical groups. Conclusion: Wearing an oronasal surgical mask did not affect the 6MWDs of patients with advanced lung diseases. Therefore, a masked 6MWT appears to provide a reliable examination of functional exercise capacity in this cohort.

(c) 2021 The Author(s)

Published by S. Karger AG, Basel

\section{Introduction}

Lung transplantation is an established treatment modality for selected patients with end-stage lung diseases. The number of transplantations reported globally has increased continuously in the past decades, to a most recently documented record of approximately 4,500 transplantations in 2016 [1].
Correspondence to:

Isabell Anna Just, ijust@dhzb.de 
The 6-minute walk test (6MWT) is a key instrument in the clinical assessment of functional exercise capacity in patients with severe pulmonary diseases. Furthermore, due to its value in predicting mortality risk, the 6-minute walk distance (6MWD) is included in lung allocation scores (LASs), which, as a whole, are based on patients' individual medical urgency and models estimating posttransplant survival and are used for the distribution of most donor lungs worldwide [2-5].

Within the scope of the recent corona virus disease (COVID-19) pandemic, preventive regulations for ambulatory care were largely intensified in accordance with instructions of local pandemic boards and recent recommendations of the European Centre for Disease Prevention and Control [6]. Particularly, a critical consideration of the necessity of each outpatient appointment and an optimization of current hygienic precautions, including the provision of surgical oronasal masks to every asymptomatic patient entering the ambulatory department, were introduced.

Especially patients with chronic respiratory insufficiency perceive oronasal masks as discomforting, increasing dyspnea sensation. The impact of masking on exercise capacity and thus on 6MWD, which might significantly influence therapeutic strategies and possible donor organ allocation, is unknown in this cohort [7]. Therefore, we retrospectively analyzed the effect of wearing an oronasal surgical mask on 6MWD in patients with stable end-stage pulmonary diseases.

\section{Materials and Methods}

\section{Study Cohort}

We retrospectively analyzed data from 64 patients with endstage lung diseases who performed a 6MWT during the COVID-19 pandemic wearing a surgical oronasal mask and who had previously performed the tests in our ambulatory department of thoracic organ transplantation in a pre-COVID-19 pandemic era without a mask. Patients with primary pulmonary vascular diseases (i.e., pulmonary arterial hypertension, chronic thromboembolic pulmonary hypertension, and World Health Organization [WHO] classes I and IV) were not included in this study. Patients with pulmonary vascular hypertension due to a chronic lung disease (WHO class III) were included in this investigation. In line with publications of the American Thoracic Society (ATS), changes of $\pm 6 \%$ in the forced vital capacity (FVC) in patients with leading fibrotic diseases and of $\pm 10 \%$ in the forced expiratory volume in the first second of expiration (FEV1) in patients with obstructive lung diseases were defined as clinically important differences in pulmonary function. Therefore, patients showing these changes in the body plethysmography between the pre-pandemic and pandemic assessment were excluded from the study $[8,9]$. For patients with cystic fibrosis, both changes in FVC and FEV1 were considered for an evaluation of clinical stability of the lung disease.

\section{Surgical Oronasal Masks}

All masks were handed out to the patients immediately before starting the tests. The hospital staff, who guided the 6MWT, supervised the correct fitting of the masks throughout the walk. The masks used were disposable, non-filtering medical masks, and not particulate respirators, as previously described in $\mathrm{WHO}$ guidelines [10]. All masks were CE-certified accompanied by an EU/EC Declaration of Conformity. The use was within the expiration dates.

\section{Data Collection}

Patients' baseline characteristics and results of the 6MWT, body plethysmography, and blood gas testing were recorded anonymously in an electronical database at 2 time points: (i) before and (ii) during the COVID-19 pandemic. The data from the pre-COVID-19 pandemic were collected between June 19, 2019 and March 12, 2020, and the data during the COVID-19 pandemic were collected between March 23, 2020 and July 3, 2020. This retrospective analysis was approved by the Ethics Committee of Charité - Universitätsmedizin Berlin (EA2/111/20).

\section{Measurements}

All measurements were performed within the scope of routine ambulatory care. The 6MWT and body plethysmography (MasterScreen Body Plethysmograph, Erich Jaeger GmbH, Würzburg, Germany) were performed according to the standards of the American Thoracic and European Respiratory Society [11]. During the 6MWT, oxygen was supplied according to the individual need by a nasal cannula, which was worn under the mask. Blood gases were analyzed in capillary blood samples that were taken after resting for at least $15 \mathrm{~min}$ in a quiet room (ABL800 FLEX; Radiometer $\mathrm{GmbH}$, Krefeld, Germany).

\section{Statistical Analysis}

Continuous data are summarized as mean and standard deviation or, in the case of skewed data, as median and interquartile range. Frequencies and percentages are reported for categorical data. Patient groups were compared using the t-test for paired samples. Equivalence tests for paired samples were used for 6MWDs with $25 \mathrm{~m}$ as an upper and $-25 \mathrm{~m}$ as a lower limit. SPSS 25 and $\mathrm{R} 4.0$ were used for statistical analysis.

\section{Results}

\section{Patient Characteristics}

Between March 23, 2020 and July 3, 2020, 64 patients with end-stage lung disease performed 6MWTs wearing an oronasal surgical mask in our ambulatory department; pulmonary function was additionally assessed by body plethysmography and blood gas analysis. In 31 patients, the clinical pulmonary function was rated as stable according to predefined minimal clinically important differences (MCIDs) in the body plethysmography assessments. Four patients were excluded due to a primary vas- 
cular disease, 29 patients due to a clinically unstable pulmonary function, and 1 patient due to a major depressive episode with fatigue and a decreased ability to perform physical activities (Fig. 1).

The median age of the 31 included patients was 55.0 (46-58) years; 15 (48\%) were male. The median BMI was 21.9 (19.1-26.2). Ten (32.2\%) patients were on the lung transplant waiting list of Eurotransplant, with a median LAS of 34.3 (31.9-36.2). Twenty (65\%) patients had chronic obstructive pulmonary diseases (COPD), 7 (23\%) had interstitial lung diseases, and 4 (13\%) had other endstage lung diseases ( 3 cystic fibrosis, 1 destroyed lung due to abscessing pneumonia).

For all evaluated patients, results of a battery of tests (6MWD without a mask, body plethysmography, and blood gases), all of which were performed in our institution in a pre-COVID-19 pandemic era, were available. The first and second ambulatory visits were separated by a median of 99 (89-134) days.

\section{Pulmonary Function}

The results of the body plethysmography of the patients with clinically stable pulmonary function are presented in Table 1 .

\section{6-Minute Walk Test}

The mean total walk distance of all patients $(n=31)$ without and with wearing a surgical oronasal mask was 306.9 (101.9) and 305.7 (103.9) $\mathrm{m}$, respectively, with a mean difference of -1.19 (95\% confidence interval -13.411.03). This difference in $6 \mathrm{MWD}$ can be regarded statistically equivalent to zero $(p<0.001)$. Also, there was no significant difference between the 3 groups (Table 2; Fig. 2).

\section{Discussion/Conclusion}

The aim of this study was to evaluate the impact of wearing an oronasal surgical mask on 6MWDs in patients with end-stage lung disease. We included patients (i) who performed the 6MWT wearing a mask during the COVID-19 pandemic and (ii) who had already performed the test unmasked during an ambulatory visit in our institution prior to the pandemic.

In order to comply with the ATS guidelines, which suggest performing walk tests on a flat, straight enclosed 30 -m corridor, $6 \mathrm{MWT}$ are routinely performed in hallways of ambulatory departments or clinical wards; patients performing the test thus inevitably pass medical

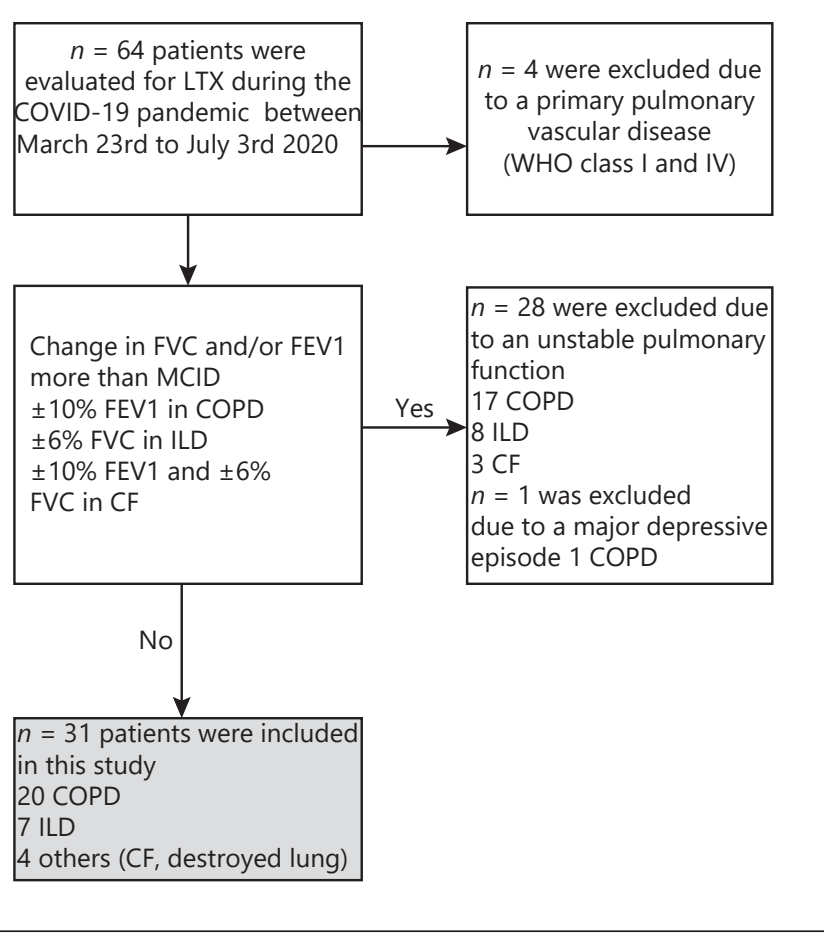

Fig. 1. Flowchart showing the selection of patients included in this study. CF, cystic fibrosis; COPD, chronic obstructive pulmonary disease; FEV1, forced expiratory volume in the first second of expiration; FVC, forced vital capacity; ILD, interstitial lung disease; MCID, minimal clinically important difference; WHO, World Health Organization; LTX, lung transplantation.

personnel and other patients [5]. Therefore, knowledge concerning the impact of a mask, which is required especially in times of pandemics to avoid a spread of infectious diseases, is incredibly valuable for caregivers assessing patients with advanced pulmonary diseases.

The majority of the patients evaluated in our study suffered from COPD (65\%) followed by interstitial lung disease (23\%) and thus represent a typical population undergoing evaluation for and on lung transplantation waiting list of Eurotransplant [1]. All patients who were found to exhibit clinically important changes in pulmonary function between the 2 investigations were excluded from the analysis in order to eliminate significant differences in $6 \mathrm{MWDs}$ due to a loss of functional exercise capacity.

In the clinically stable cohort, a comparison of $6 \mathrm{MWDs}$ when wearing a surgical mask to the results of the previously unmasked 6MWDs revealed a change of $-1.2 \mathrm{~m}$ $(-13.4$ to 11.03$)$. Statistical equivalence could be confirmed if $p<0.001$. The definition of MCID in 6MWDs in patients with pulmonary diseases has not been applied 
Table 1. Pulmonary function of patients with stable end-stage lung diseases

\begin{tabular}{|c|c|c|c|c|c|c|}
\hline & \multicolumn{3}{|c|}{ Before the COVID-19 pandemic } & \multicolumn{3}{|c|}{ During the COVID-19 pandemic } \\
\hline FEV1, L mean (SD) & $0.76(0.27)$ & $2.32(1.01)$ & $0.8(0.16)$ & $0.77(0.27)$ & $2.33(1.01)$ & $0.80(0.14)$ \\
\hline FEV1/FVC, \% mean (SD) & $41.88(13.90)$ & $86.14(9.79)$ & $51.14(10.79)$ & $39.42(1.62)$ & $85.16(10.54)$ & $46.20(3.89)$ \\
\hline TLC, L mean (SD) & $7.30(2.19)$ & $4.18(2.01)$ & $5.54(1.71)$ & $7.36(2.19)$ & $4.32(2.03)$ & $5.50(1.59)$ \\
\hline Oxygen, L/min median (IQR) & $0.5(0-2)$ & $2(0-2)$ & $0.5(0-1.75)$ & $1(0-2)$ & $2(0-2)$ & $1.5(1-2.75)$ \\
\hline $\mathrm{pO}_{2}, \mathrm{~mm} \mathrm{Hg}$ median (IQR) & $66.0(62.5-71.0)$ & $71.0(61-74)$ & $63.0(57.2-66.5)$ & $76.0(65.0-94.0)$ & $73.0(66.0-103.0)$ & $90.0(76.75-100.25)$ \\
\hline $\mathrm{pCO}_{2}, \mathrm{~mm} \mathrm{Hg}$ median (IQR) & $43.5(38.3-49.8)$ & $36.0(35.0-42.0)$ & $43.0(38$. to 56.3$)$ & $44.0(39.3-52.0)$ & $36.0(34.0-43.0)$ & $42.5(36.8-47.5)$ \\
\hline $\mathrm{pH}$ median (IQR) & $7.42(7.40-7.44)$ & $7.43(7.42-7.46)$ & $7.41(7.38-7.47)$ & $7.42(7.37-7.44)$ & $7.42(7.41-7.44)$ & $7.41(7.39-7.44)$ \\
\hline $\mathrm{HCO}_{3}{ }^{-}, \mathrm{mmol} / \mathrm{L}$ median (IQR) & $28.0(26.0-32.0)$ & $25.0(26.0-28.0)$ & $30.0(25.3-34.0)$ & $28.5(25.3-32.8)$ & $25.0(24.0-27.0)$ & $25.5(24.5-30.5)$ \\
\hline
\end{tabular}

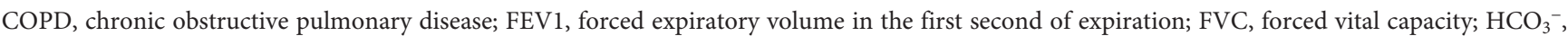
bicarbonate; ILD, interstitial lung disease; $\mathrm{pCO}_{2}$, partial pressure of carbon dioxide; $\mathrm{pO}_{2}$, partial pressure of oxygen; $R$, resistance; $\mathrm{RV}$, residual volume; TLC, total lung capacity; IQR, interquartile range.

Table 2. $6 \mathrm{MWT}$ of patients with stable end-stage lung diseases without and with wearing a mask

\begin{tabular}{|c|c|c|c|c|}
\hline Oxygen supply during 6MWT, L/min mean (SD) & $1.95(1.6)$ & $2.01(1.5)$ & $0.06(-0.15$ to 0.27$)$ & $<0.001$ \\
\hline \multicolumn{5}{|l|}{$6 M W D$, m mean $(S D)$} \\
\hline All $(n=31)$ & $306.9(101.9)$ & $305.7(103.8)$ & $-1.19(-13.4$ to 11.0$)$ & $<0.001$ \\
\hline Others $(n=4)$ & $389.5(44.2)$ & $387.0(23.8)$ & $-2.50(-36.6$ to 31.6$)$ & - \\
\hline \multicolumn{5}{|c|}{ Oxygen saturation at maximum exercise, \% mean (SD) } \\
\hline All $(n=31)$ & $88.3(8.0)$ & $89.0(7.1)$ & $0.06(-1.4$ to 2.8$)$ & 0.003 \\
\hline $\operatorname{COPD}(n=20)$ & $89.6(6.2)$ & $90.0(7.0)$ & $0.40(-1.3$ to 2.1$)$ & 0.003 \\
\hline $\operatorname{ILD}(n=7)$ & $83.3(44.2)$ & $84.3(23.8)$ & $1.00(-8.4$ to 10.4$)$ & - \\
\hline
\end{tabular}

The $t$ tests were not performed due to limited validity in the groups with small sample sizes. CI, confidence interval; COPD, chronic obstructive pulmonary disease; ILD, interstitial lung disease; SD, standard deviation; 6MWD, 6-minute walk distance; 6MWT, 6-minute walk test.

consistently in the current body of the literature, mainly due to the gradual evolution of corresponding clinical research. However, the threshold of $54 \mathrm{~m}$ for patients with COPD, which was proposed by Redelmeier et al. [12], has been rectified to $25-43 \mathrm{~m}$ for COPD and $24-45 \mathrm{~m}$ for idiopathic pulmonary fibrosis according to the latest clinical trials $[10,11,13,14]$. Thus, we considered a threshold of $25 \mathrm{~m}$ as a margin of clinical relevance. The lower and upper limits of the $95 \%$ confidence interval of differences in $6 \mathrm{MWD}$ when wearing a mask were -13.4 and $+11.0 \mathrm{~m}$ and thus clearly less than the MCID (Fig. 1a).

Based on this, we conclude that wearing an oronasal surgical mask does not appear to affect 6MWDs in patients with end-stage lung diseases. This is in line with the results of a previous clinical trial by Person et al. [15], who found no impact of wearing a surgical mask 


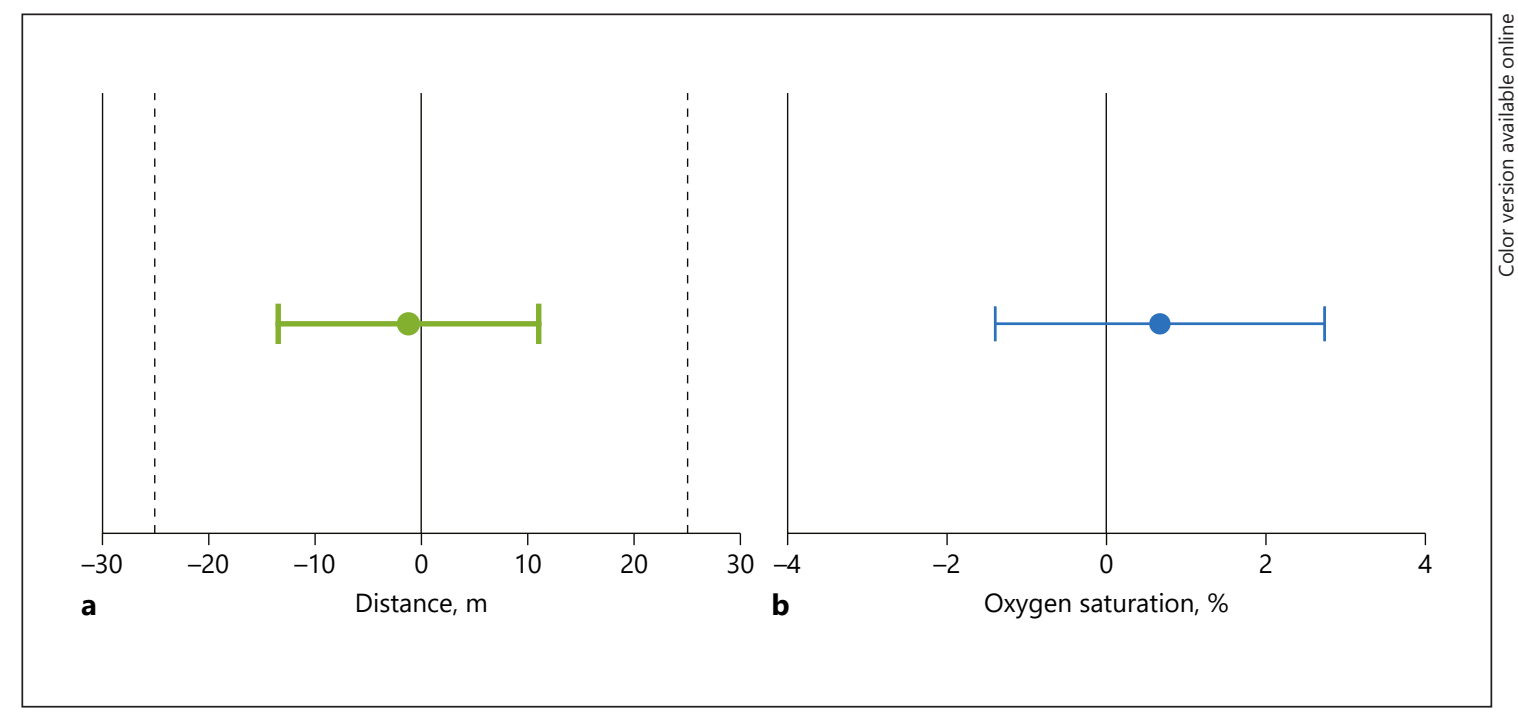

Fig. 2. Differences in 6MWD (a) and oxygen saturation at maximum exercise during the 6MWT (b) with or without wearing an oronasal surgical mask in all patients (mean, 95\% CI). 6MWT, 6-minute walk test; 6MWD, 6-minute walk distance; CI, confidence interval.

on walked distances of 44 healthy subjects performing the 6MWT. However, taking into account that patients with advanced pulmonary diseases - like those in our special cohort - have reduced tidal volumes compensated by higher respiratory rates, a single breath might be less affected by the suction of the mask than healthy individuals with greater tidal volumes. This hypothesis is supported by the findings of Fikenzer et al. who identified a reduction of spirometry lung function parameters (FVC, FEV1, and peak expiratory flow) by wearing a mask in 12 active and healthy young male individuals. Interestingly, in this cohort, wearing a surgical mask also showed a decrease in exercise performance, in terms of maximum power and maximum oxygen uptake, measured by an ergometry test. A noteworthy aspect, which the authors point out, is the fact that the surgical masks, other as in our 6MWT, were completely sealed by with spirometry mask, not allowing leakages to eyes and ears [16].

Overall, we postulate that 6MWDs assessed while wearing an oronasal mask are valid in patients with advanced lung diseases and sufficiently reflect disease severity. Consequently, masked 6MWTs may be used for assessing the functional exercise capacity in patients with advanced pulmonary disease, as is needed when evaluating the LAS, during the COVID-19 pandemic. Whether these results are transferable to other clinical indications for the 6MWT, for example, cardiovascular diseases, remains elusive $[17,18]$. However, further research in pro- spective, controlled clinical trials is necessary to confirm our findings and for further investigations in other clinical settings.

\section{Limitations}

It is to be acknowledged that this study has certain limitations: the investigation was performed retrospectively in a single center with a limited sample size, analyzing only surgical masks (not particulate respirators).

\section{Statement of Ethics}

The authors are accountable for all aspects of the work in ensuring that questions related to the accuracy or integrity of any part of the work are appropriately investigated and resolved. The trial was conducted in accordance with the Declaration of Helsinki (as revised in 2013). The data analysis was approved by the Ethics Committee of Charité - Universitätsmedizin Berlin (EA2/111/20). Due to its retrospective nature, no informed consent was required.

\section{Conflict of Interest Statement}

Felix Schoenrath reports nonfinancial support from Medtronic, grants from Novartis, grants from Abbott, and personal fees from Cardiorentis, outside the submitted work. Volkmar Falk has relevant financial activities outside the submitted work with the commercial entities in relation to educational grants (incl. travel support), fees for lectures and speeches, fees for professional consultation, fees for research, and study funds. The remaining authors have no conflicts of interest to declare. 


\section{Funding Sources}

This research received no specific grant from any funding agency in the public, commercial, or not-for-profit sectors.

\section{Author Contributions}

(I) Conception and design: Just and Knierim. (II) Administrative support: Passinger. (III) Provision of study materials or patients: Falk, Just, Kemper, Knierim, Knosalla, and Passinger. (IV) Collection and assembly of data: Just, Knierim, and Passinger. (V) Data analysis and interpretation: Falk, Just, Kemper, Knierim, Knosalla, Schoenrath, and Stein. (VI) Manuscript writing: all authors. (VII) Final approval of manuscript: all authors.

\section{References}

1 Chambers DC, Cherikh WS, Harhay MO, Hayes D Jr, Hsich E, Khush KK, et al. The International Thoracic Organ Transplant Registry of the International Society for Heart and Lung Transplantation: Thirty-sixth adult lung and heart-lung transplantation Report-2019; Focus theme: Donoer and recipient size match. J Heart Lung Transplant. 2019;38:1042-55.

2 Dajczman E, Wardini R, Kasymjanova G, Préfontaine D, Baltzan MA, Walkovers N. Six minute walk distance is a predictor of survival in patients with chronic obstructive pulmonary disease undergoing pulmonary rehabilitation. Can Respir J. 2015;22(4):225-9.

3 du Bois RM, Albera C, Bradford WZ, Costabel U, Leff JA, Noble PW, et al. 6-minute walk distance is an independent predictor of mortality in patients with idiopathic pulmonary fibrosis. Eur Respir J. 2014;43(5):1421-9.

4 Gottlieb J. Lung allocation. J Thorac Dis. 2017;9(8):2670-4.

5 Crapo RO. ATS statement: guidelines for the six-minute walk test. Am J Respir Crit Care Med. 2002;166(1):111-7.

6 European Centre for Disease Prevention and Control. Personal protective equipment (PPE) needs in healthcare settings for the care of patients with suspected or confirmed 2019nCoV. Stockholm: ECDC; 2020. Available from: https://www.ecdc.europa.eu/en/publications-data/personal-protective-equipment-ppe-needs-healthcare-settings-carepatients.
7 Weiss MM, Weiss PD, Weiss DE, Weiss JB. Disrupting the transmission of influenza A: face masks and ultraviolet light as control measures. Am J Public Health. 2007;97(Suppl 1):S32-7.

8 Behr J. A small change in FVC but a big change for IPF: defining the minimal clinically important difference. Am J Respir Crit Care Med. 2011;184(12):1329-30.

9 Jones PW, Beeh KM, Chapman KR, Decramer M, Mahler DA, Wedzicha JA. Minimal clinically important differences in pharmacological trials. Am J Respir Crit Care Med. 2014;189(3):250-5.

10 Infection prevention and control of epidemic- and pandemic-prone acute respiratory infections in health care. Geneva: World Health Organization; 2014.

11 Wanger J, Clausen JL, Coates A, Pedersen OF, Brusasco V, Burgos F, et al. Standardisation of the measurement of lung volumes. Eur Respir J. 2005;26:511-22.

12 Redelmeier DA, Bayoumi AM, Goldstein RS, Guyatt GH. Interpreting small differences in functional status: the Six Minute Walk test in chronic lung disease patients. Am J Respir Crit Care Med. 1997;155(4):1278-82.
13 Polkey MI, Spruit MA, Edwards LD, Watkins ML, Pinto-Plata V, Vestbo J, et al. Six-minutewalk test in chronic obstructive pulmonary disease: minimal clinically important difference for death for hospitalization. Am J Respir Crit Care Med. 2013;187:382-6.

14 du Bois RM, Weycker D, Albera C, Bradford WZ, Costabel U, Kartashov A, et al. Six-minute-walk test in idiopathic pulmonary fibrosis: test validation and minimal clinically important difference. Am J Respir Crit Care Med. 2011;183(9):1231-7.

15 Person E, Lemercier C, Royer A, Reychler G. [Effect of a surgical mask on six minute walking distance]. Rev Mal Respir. 2018;35(3): 264-8.

16 Fikenzer S, Uhe T, Lavall D, Rudolph U, Falz $\mathrm{R}$, Busse M, et al. Effects of surgical and FFP2/ N95 face masks on cardiopulmonary exercise capacity. Clin Res Cardiol. 2020;109(12): 1522-30.

17 O'Keeffe ST, Lye M, Donnellan C, Carmichael DN. Reproducibility and responsiveness of quality of life assessment and six minute walk test in elderly heart failure patients. Heart. 1998;80(4):377-82.

18 Bittner V. Six-minute walk test in patients with cardiac dysfunction. Cardiologia. 1997; 42(9):897-902. 